\title{
Severe myocardial ischemia in a patient with diabetes mellitus and left bundle branch block
}

\author{
Brittain Heindl, $M D,{ }^{a}$ Ami E. Iskandrian, MD, MASNC, ${ }^{a}$ \\ and Fadi G. Hage, MD, MASNC ${ }^{\mathrm{a}, \mathrm{b}}$ \\ a Division of Cardiovascular Disease, University of Alabama at Birmingham, Birmingham, AL \\ b Section of Cardiology, Birmingham Veterans Affairs Medical Center, Birmingham, AL
}

Received Apr 15, 2019; accepted May 29, 2019

doi: $10.1007 / \mathrm{s} 12350-019-01790-0$

We present the case of a 69-year-old woman with diabetes mellitus, left bundle branch block, and obesity who develops cardiogenic shock after undergoing myocardial perfusion imaging and diagnostic left heart catheterization, but prior to coronary revascularization. This case highlights the risk of cardiac decompensation awaiting surgery in patients with multivessel coronary artery disease. It also emphasizes the subtleties involved in imaging patients with diabetes mellitus and left bundle branch block, as well as the risk of developing complete heart block in the setting of ischemia with underlying conduction disease. (J Nucl Cardiol 2021;28:278-88.)

Key Words: Diabetes $\cdot$ cardiogenic shock $\cdot$ obesity $\cdot$ SPECT $\cdot$ perfusion imaging

\begin{tabular}{|llll|}
\hline Abbreviations & LVH & Left ventricular hypertrophy \\
DM & Diabetes mellitus & RBBB & Right bundle branch block \\
ECG & Electrocardiogram & RV & Right ventricular \\
EF & Ejection fraction & TTE & Transthoracic echocardiogram \\
LBBB & Left bundle branch block & & \\
LV & Left ventricular & & \\
\hline
\end{tabular}

\section{CASE PRESENTATION}

A 69-year-old woman with a history of type 2 diabetes mellitus (DM), hypertension, class 3 obesity, and dyslipidemia presented to an urgent care clinic with exertional dyspnea that steadily worsened over the last 5 months. She denied orthopnea but described paroxysmal nocturnal dyspnea for which she turned on the

Electronic supplementary material The online version of this article (https://doi.org/10.1007/s12350-019-01790-0) contains supplementary material, which is available to authorized users.

Reprint requests: Brittain Heindl, MD, Division of Cardiovascular Disease, University of Alabama at Birmingham, Birmingham, AL; bfheindl@uabmc.edu

J Nucl Cardiol

$1071-3581 / \$ 34.00$

Copyright (C) 2019 American Society of Nuclear Cardiology. ceiling fan or walked outside to help with breathing. She denied having any chest pain.

Four months prior to this visit she presented to the emergency department (ED) with the above symptoms. Evaluation was negative and she was discharged without intervention. Between then and her current visit she was evaluated in the ED at multiple facilities five times with similar symptoms. She was seen by both otolaryngology and pulmonary medicine in the interim. She underwent naso-laryngoscopy and was eventually prescribed albuterol and fluticasone-vilanterol inhalers. Despite these interventions, her exertional dyspnea continued to worsen.

She has no prior history of atherosclerotic cardiovascular disease (CVD). She reports having a pharmacologic stress test 8 months prior to this presentation which has been described to her as normal. Her surgical history is limited to a parotid gland removal in 


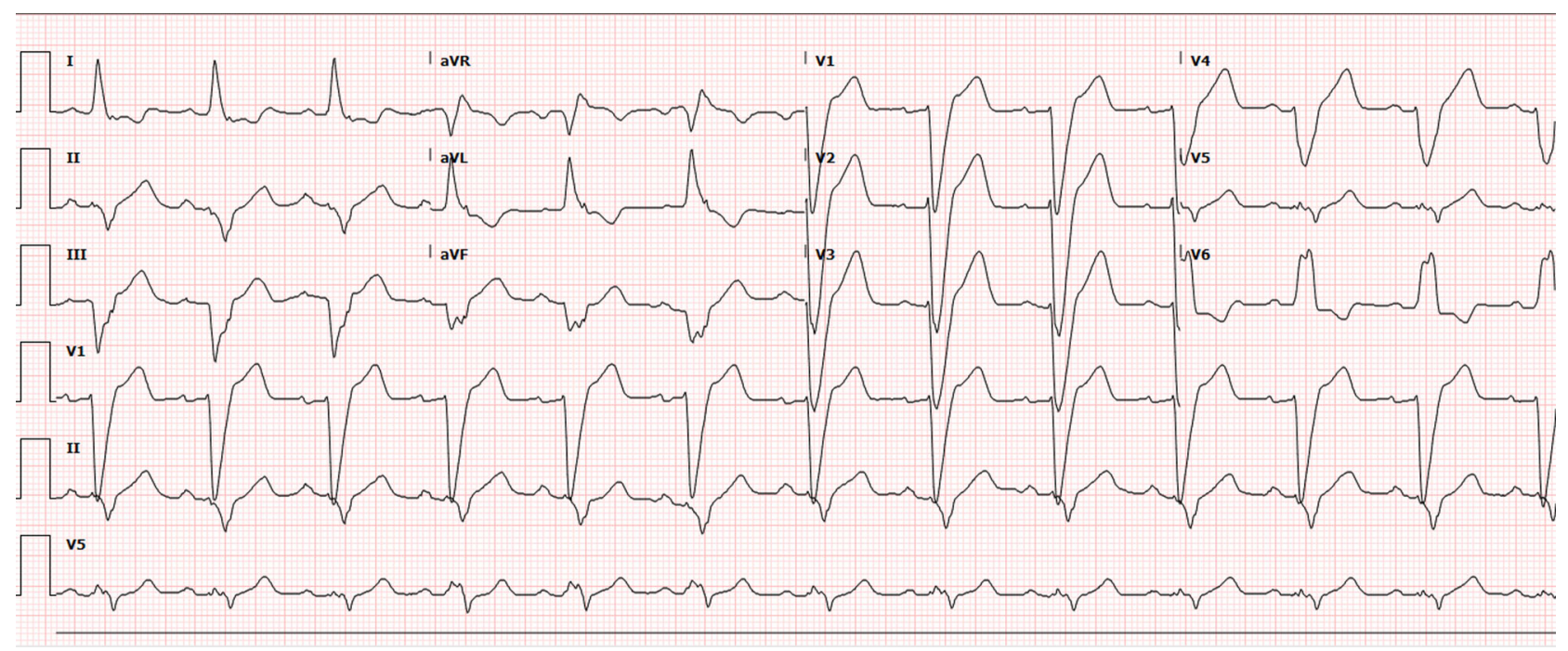

Figure 1. 12-Lead ECG showing normal sinus rhythm and LBBB.

Table 1. Initial laboratory values

\begin{tabular}{ll} 
Chemistry & \\
Sodium & $140 \mathrm{mMol} / \mathrm{L}$ \\
Potassium & $3.6 \mathrm{mMol} / \mathrm{L}$ \\
Chloride & $101 \mathrm{mMol} / \mathrm{L}$ \\
Bicarbonate & $28 \mathrm{mMol} / \mathrm{L}$ \\
BUN & $37 \mathrm{mg} / \mathrm{dL}$ \\
Creatinine & $1.3 \mathrm{mg} / \mathrm{dL}$ \\
Glucose & $151 \mathrm{mg} / \mathrm{dL}$ \\
Calcium & $9.5 \mathrm{mg} / \mathrm{dL}$ \\
Protein & $7.3 \mathrm{~g} / \mathrm{dL}$ \\
Albumin & $3.9 \mathrm{~g} / \mathrm{dL}$ \\
Liver profile & \\
Total bilirubin & $0.4 \mathrm{mg} / \mathrm{dL}$ \\
Alk Phos & $69 \mathrm{U} / \mathrm{L}$ \\
ALT & $21 \mathrm{U} / \mathrm{L}$ \\
AST & $18 \mathrm{U} / \mathrm{L}$ \\
Cardiac markers & $180 \mathrm{pg} / \mathrm{mL}$ \\
BNP & $<0.030 \mathrm{ng} / \mathrm{mL}^{2}$ \\
Troponin-I & $8.25 \times 10^{3} \mathrm{~mm}^{3}$ \\
Hematology & $13.3 \mathrm{~g} / \mathrm{dL}$ \\
WBC & $39 \mathrm{~g} / \mathrm{dL}$ \\
Hgb & $173.6 \times 10^{3} \mathrm{~mm}^{3}$ \\
HCT & \\
Platelet & $136 \mathrm{mg} / \mathrm{dL}$ \\
Lipid profile & $199 \mathrm{mg} / \mathrm{dL}$ \\
Total cholesterol & $37 \mathrm{mg} / \mathrm{dL}$ \\
Triglycerides & $67 \mathrm{mg} / \mathrm{dL}$ \\
HDL & $7.2 \%$ \\
LDL & \\
Hemoglobin A $1 \mathrm{c}$ & \\
\hline
\end{tabular}

1980, due to an underlying tooth infection. She smoked very briefly ( $<1$ year) in the 1980s. She denied alcohol or illicit substance use. Her family history is significant for DM and kidney disease in her mother and a central nervous system malignancy in her father. Her only sibling is alive and healthy. She does not have any significant family history of CVD.

Home medications include aspirin $81 \mathrm{mg}$ daily, hydrochlorothiazide-losartan $12.5-50 \mathrm{mg}$ daily, insulin glargine 50 units subcutaneously twice daily, metformin $1000 \mathrm{mg}$ twice daily, and carvedilol $25 \mathrm{mg}$ twice daily.

On arrival to the urgent care clinic, her heart rate was $76 \mathrm{bpm}$ and regular, respiratory rate $18 / \mathrm{min}$, blood pressure $157 / 83 \mathrm{mmHg}$, oxygen saturation $97 \%$ on room air, and body mass index $47 \mathrm{~kg} / \mathrm{m}^{2}$. Physical exam was otherwise unremarkable, without clear signs of heart failure. An electrocardiogram (ECG) was obtained demonstrating normal sinus rhythm with left bundle branch block (LBBB, Fig. 1). Chest X-ray (CXR) was unremarkable.

Since the chronicity of the LBBB was unknown, the patient was transferred to the ED to rule out acute coronary syndrome. In the ED and upon subsequent chart review, the patient's LBBB was noted to be present during her ED presentation 4 months earlier. Her laboratory values are shown in Table 1 . The patient was admitted to the medicine service for further evaluation.

The following morning a regadenoson single-photon emission computed tomography (SPECT) myocardial perfusion imaging (MPI) was performed. The images from this study are shown in Fig. 2 and demonstrate a large perfusion abnormality of moderate 
A



B

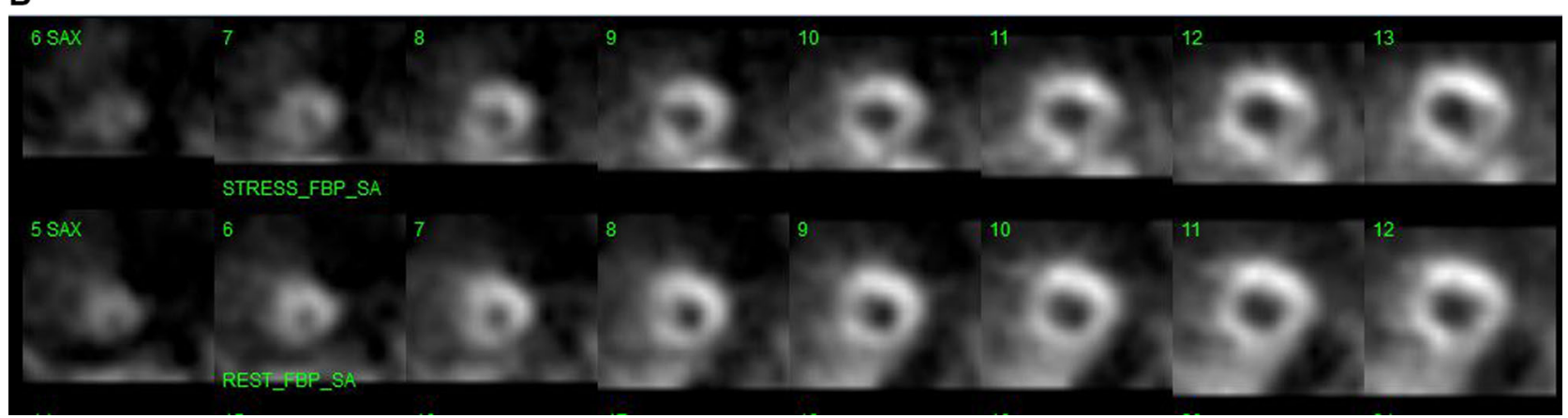

Figure 2. (A) SPECT stress/rest images in 3 projections showing a large perfusion abnormality of moderate severity in 3-vessel distribution. The 7 segments involved are the apex, distal septal, distal lateral, distal inferior, mid- and basal inferolateral, and mid-inferior. There is considerable reversibility. (B) Gray scale images demonstrate transient ischemic dilation. (C) The stress, rest, and reversibility polar maps demonstrate a large perfusion defect with significant reversibility. The abnormality involves $48 \%$ of LV myocardium. (D) LVEF by gated SPECT was 24 post stress and $33 \%$ post rest (Video in Online Supplement). The end-systolic frames are shown for the stress study on the left and the rest study on the right. 




D

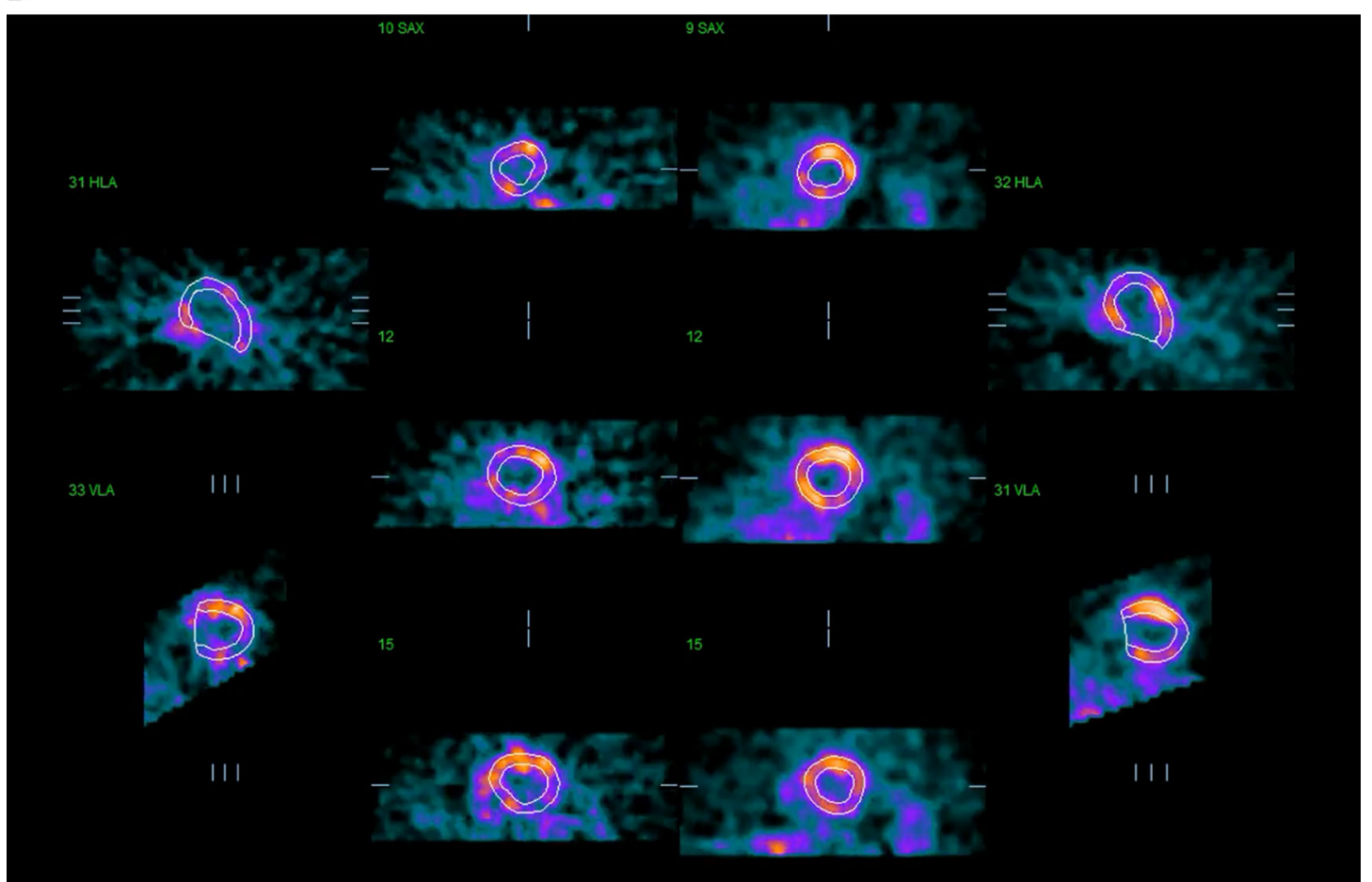

Figure 2. continued. 
A



B

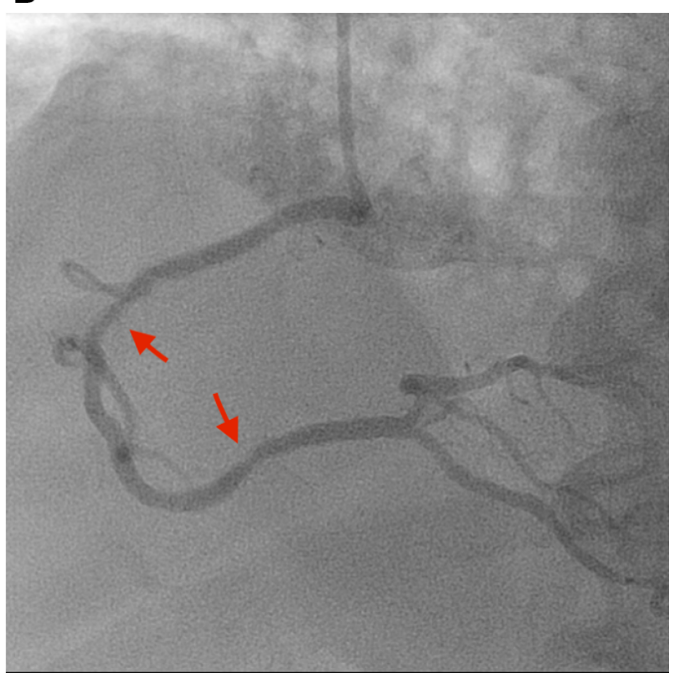

Figure 3. Coronary angiogram showing $70 \%$ left main stenosis, $80 \%$ ostial stenosis of the ramus intermedius, $60 \%$ proximal LAD stenosis, $70 \%$ stenosis of a large diagonal branch (A), and $60 \%$ stenosis of the mid-RCA $(\mathbf{B})$.

Table 2. Pressure measurements obtained via RHC

\begin{tabular}{ll}
\hline Mean right atrial pressure & $13(\mathrm{mmHg})$ \\
Right ventricular pressures & $43 / 12(\mathrm{mean} \mathrm{20})$ \\
Pulmonary artery pressures & $54 / 32(39)(\mathrm{mmHg})$ (saturation $50 \%)$ \\
Pulmonary vascular resistance & $361 \mathrm{dyne} \cdot \mathrm{s} \cdot \mathrm{cm}^{-5}$ \\
Mean pulmonary wedge pressure & $18(\mathrm{mmHg})$ \\
Aortic pressures & $161 / 94(119)(\mathrm{mmHg})($ Saturation $94 \%)$ \\
Systemic vascular resistance & $1820 \mathrm{dyne} \cdot \mathrm{s} \cdot \mathrm{cm}^{-5}$ \\
Cardiac index (Fick) & $1.67 \mathrm{~L} / \mathrm{min} / \mathrm{m}^{2}$ \\
\hline
\end{tabular}

severity in the septum, apex, inferior wall, and lateral wall with significant reversibility. The abnormality involved $48 \%$ of the left ventricular (LV) myocardium. There was severe LV dysfunction with transient ischemic dilation and post stress stunning. The LV ejection fraction $[\mathrm{EF}]$ was $24 \%$ post stress (EDV 157, ESV 119) and 33\% post rest (EDV 175, ESV 118).

She was started on metoprolol $100 \mathrm{mg}$ extended release and left heart catheterization was performed the following morning. Images from her coronary angiograms are shown in Fig. 3 which revealed a 70\% left main (LM) stenosis, $80 \%$ ostial stenosis of a large ramus intermedius, a long $60 \%$ proximal stenosis of a large left anterior descending artery (LAD), and a 70\% stenosis of large diagonal branch. The left circumflex artery was non-dominant with a $50-60 \%$ stenosis of a large proximal obtuse marginal branch. The right coronary artery (RCA) had a mid $60 \%$ stenosis. LV end-diastolic pressure was $17 \mathrm{mmHg}$. Cardiovascular surgery evaluated the patient and scheduled surgical revascularization with coronary artery bypass grafting (CABG) in 2 days.

The evening after left heart catheterization, she developed acute dyspnea which was more severe than prior episodes. A transthoracic echocardiogram (TTE) showed mild concentric LV hypertrophy (LVH) with moderate to severe global hypokinesis (LVEF of 30$35 \%$, Fig. 4). Right ventricular (RV) function was mildly reduced. Mild to moderate mitral regurgitation was present with calcified mitral apparatus.

That evening the patient's clinical status quickly and unexpectedly deteriorated and she developed hypoxemia $\left(\mathrm{O}_{2}\right.$ peripheral saturation 60\%) and respiratory distress. The rapid response team was called and as she was being prepared for transport to the intensive care unit she became unresponsive and her peripheral pulse was lost (pulseless electrical activity (PEA) on the monitor). Advanced cardiovascular life support 
A

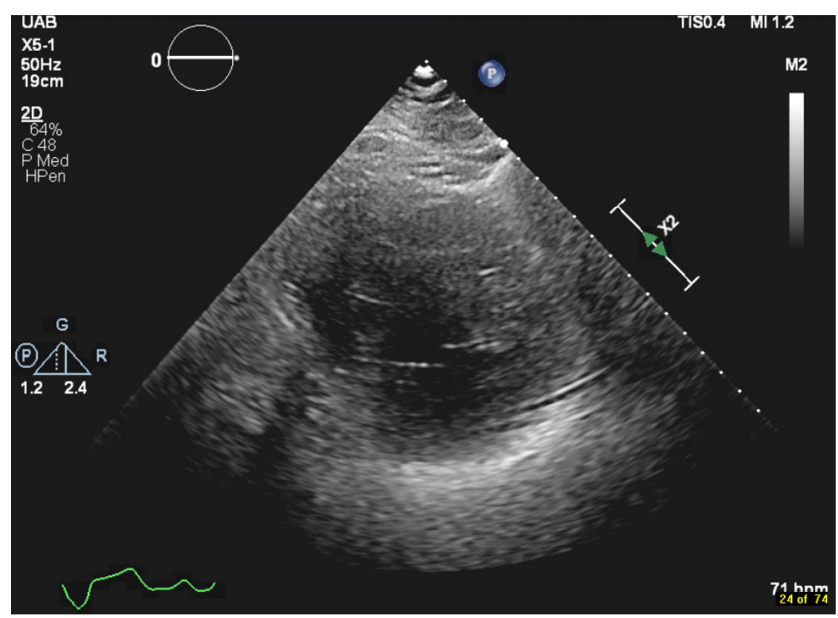

B



C

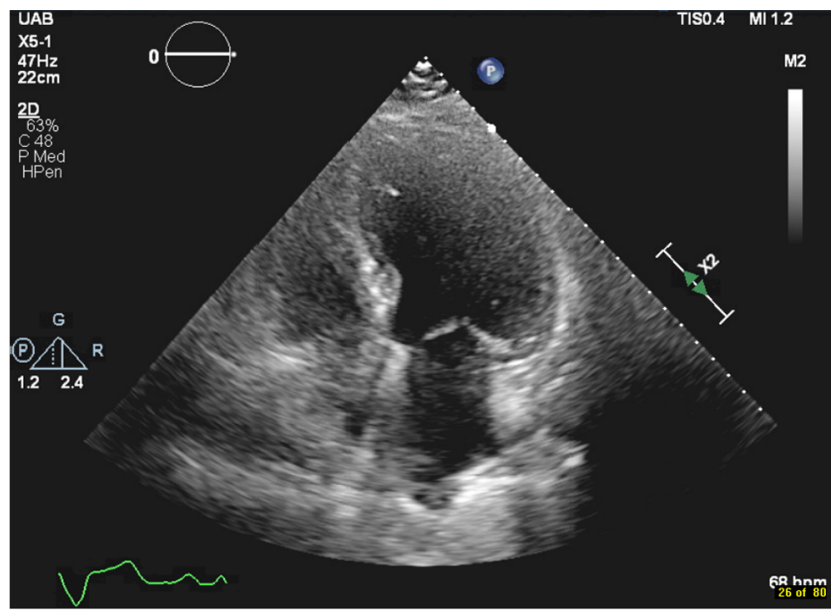

D

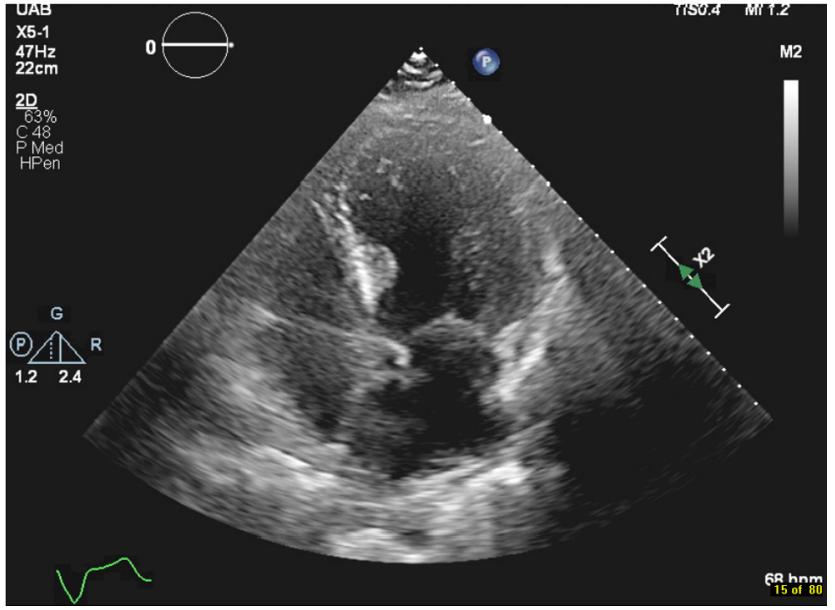

Figure 4. Echocardiogram images showing LVH with an LVEF of 30-35\%. (A, B) Short-axis views. (C, D) Apical 4 chamber views. (A, C) End-diastolic frames. (B, D) End-systolic frames.

measures were initiated and she received 6 rounds of chest compressions and 4 doses of epinephrine, and was successfully intubated. She never developed a rhythm amenable to defibrillation. Subsequently, return of spontaneous circulation (ROSC) was achieved and she was moved to the intensive care unit.

Subsequent labs revealed a lactic acid of $16 \mathrm{mmol} /$ $\mathrm{L}$ and troponin of $0.340 \mu \mathrm{g} / \mathrm{L}$. Arterial blood gas was obtained with $\mathrm{pH}$ 6.93, $\mathrm{PaCO}_{2} 64 \mathrm{mmHg}, \mathrm{PaO}_{2}$ $47 \mathrm{mmHg}$, and $\mathrm{HCO} 313 \mathrm{mEq} / \mathrm{L}$. Epinephrine was infused for persistent hypotension. CXR showed diffuse bilateral infiltrates consistent with pulmonary edema (Fig. 5).

Serial ECGs demonstrated complete heart block (CHB) with idio-ventricular rhythm, right bundle branch (RBBB) morphology, and dynamic ST changes (Fig. 6). Atropine was administered but her heart rate failed to improve. She was therefore paced using transcutaneous pacing pads at a rate of $60 \mathrm{bpm}$ with difficulty in capturing due to her large body habitus. She was started on a dopamine infusion (in addition to epinephrine). With these interventions her blood pressure improved but she still required $100 \%$ inspired oxygen with a positive end-expiratory pressure of $22 \mathrm{~cm}$ of water.

After discussion with interventional cardiology, the decision was made to proceed with emergent percutaneous coronary intervention (PCI), intra-aortic balloon pump (IABP) placement, and transvenous pacemaker placement. Central venous and arterial lines were placed. She was started on a heparin infusion. Dopamine and epinephrine were weaned and replaced with norepinephrine and dobutamine. At that point, the patient's native heart rate exceeded that of the transcutaneous pacer and pacing was stopped (Fig. 6C). 




Figure 5. CXR showing diffuse bilateral infiltrates consistent with pulmonary edema.

In the cardiac catheterization laboratory, right heart catheterization (RHC) was initially performed (Table 2). When the Swan-Ganz catheter reached the pulmonary artery, the patient developed CHB again. A temporary transvenous pacemaker was quickly placed.

Intravascular ultrasound (IVUS) demonstrated a LM minimal lumen area (MLA) of $10.1 \mathrm{~mm}^{2}$, ostial LAD MLA of $6.1 \mathrm{~mm}^{2}$, proximal LAD MLA less than $3 \mathrm{~mm}^{2}$, and distal vessel reference diameter of 3.25$3.5 \mathrm{~mm}^{2}$ (Fig. 7). Drug eluting stents were placed in the first diagonal and LAD bifurcation as well as in the midRCA. Following stent placement an IABP was inserted for additional hemodynamic support.

Upon returning to the cardiac care unit after PCI, she developed sustained ventricular tachycardia (VT) for which amiodarone infusion was initiated with restoration of sinus rhythm. She subsequently also developed a groin hematoma associated with a significant drop in hematocrit (from $14 \%$ to $8 \%$ ) for which a FemoStop ${ }^{\circledR}$ device was placed. She was transfused a total of 3 units of packed red blood cells. CT scan of the abdomen and pelvis did not reveal a retroperitoneal hematoma. Her platelet count concomitantly dropped and heparin-induced thrombocytopenia (HIT) antibody was sent. Anticoagulation was stopped and the IABP was placed at $1: 1$ counter pulsation. Bivalirudin was considered but deferred until the HIT antibody returned.

Over the course of the following day dobutamine and norepinephrine were weaned and then discontinued. She had no additional episodes of VT. Her ventilator oxygen requirements concomitantly dropped and her mental status improved and she was successfully extubated. HIT antibody returned negative and her hemoglobin concentration stabilized. Heparin was resumed and the balloon pump was transitioned to $2: 1$ counter pulsation and then later removed. With no further episodes of $\mathrm{CHB}$, the transvenous pacer was removed, along with the Swan-Ganz catheter.

Repeat TTE revealed an improved LVEF at 40$45 \%$, mild septal hypokinesis, and septal motion consistent with her underlying conduction abnormality. RV function remained mildly reduced. She was discharged to a subacute nursing facility on minimal oxygen with plans for outpatient permanent pacemaker placement.

\section{DISCUSSION}

This case emphasizes the importance of aggressively evaluating patients with DM who present with atypical symptoms and/or unexplained ECG abnormalities. It also highlights the increased risk of adverse outcomes in patients awaiting coronary revascularization after being diagnosed with severe disease. This discussion will elaborate on a few points pertinent to this case. We will discuss when to perform stress MPI in DM patients, how current imaging techniques are limited by presence of a $\mathrm{LBBB}$, and who is at risk for cardiac events in anticipation of CABG.

\section{Imaging in Patients with DM}

CVD is the leading cause of mortality in patients with DM, and these patients have a 2- to 4-fold increased risk for having CVD events, are more likely to have multivessel disease, and are more likely to develop heart failure. ${ }^{1} \mathrm{DM}$ patients have a much higher chance (up to $26 \%$ ) of having silent ischemia on stress imaging compared to individuals without DM. ${ }^{2-4}$ As in other patient groups, having moderate to severe defects on MPI predicts high risk of future CVD events. 5,6

The role of screening asymptomatic patients with DM using stress MPI has been evolving, but it is probably still true that the screening threshold is lower than non-diabetic patients. ${ }^{7}$ Specific subgroups of patients may benefit from screening: those who have had DM for a long duration (especially if not well controlled), present with atypical symptoms (specifically dyspnea), have additional risk factors, ECG changes ( $Q$ waves), peripheral arterial disease, and carotid disease. $^{5-7}$ The event rate in DM patients with dyspnea is twice that of those with chest pain. ${ }^{8}$ Multivessel perfusion abnormalities portend the worst outcomes. ${ }^{9}$ Patients with high-risk MPI, even if asymptomatic, benefit from surgical revascularization over medical management and PCI. ${ }^{10}$ This patient appropriately underwent stress imaging at an outside hospital 8 months before her current presentation, but those images were not available to us, and it is unclear how they compared to the images taken at our institution. When her dyspnea persisted and 
A

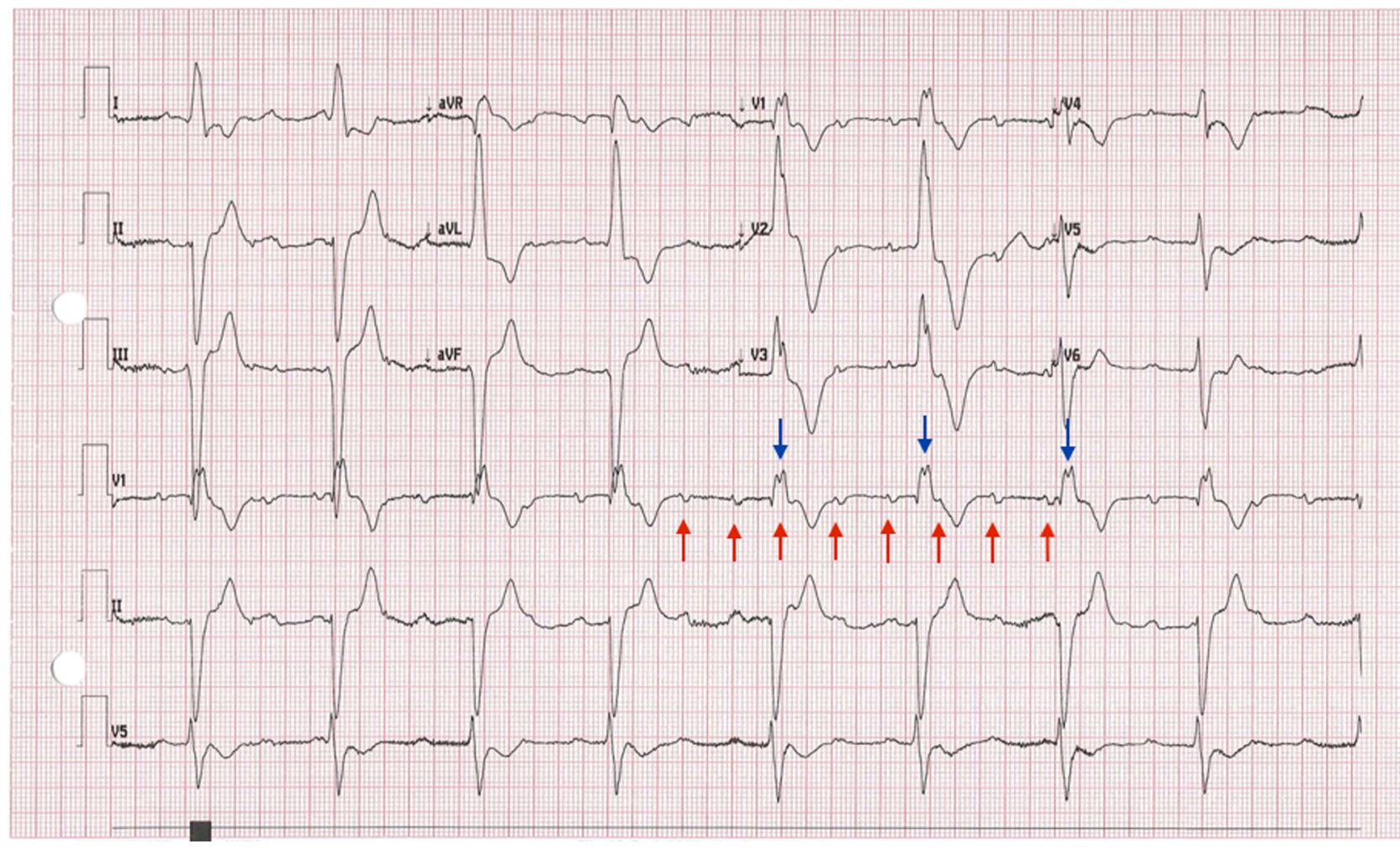

B

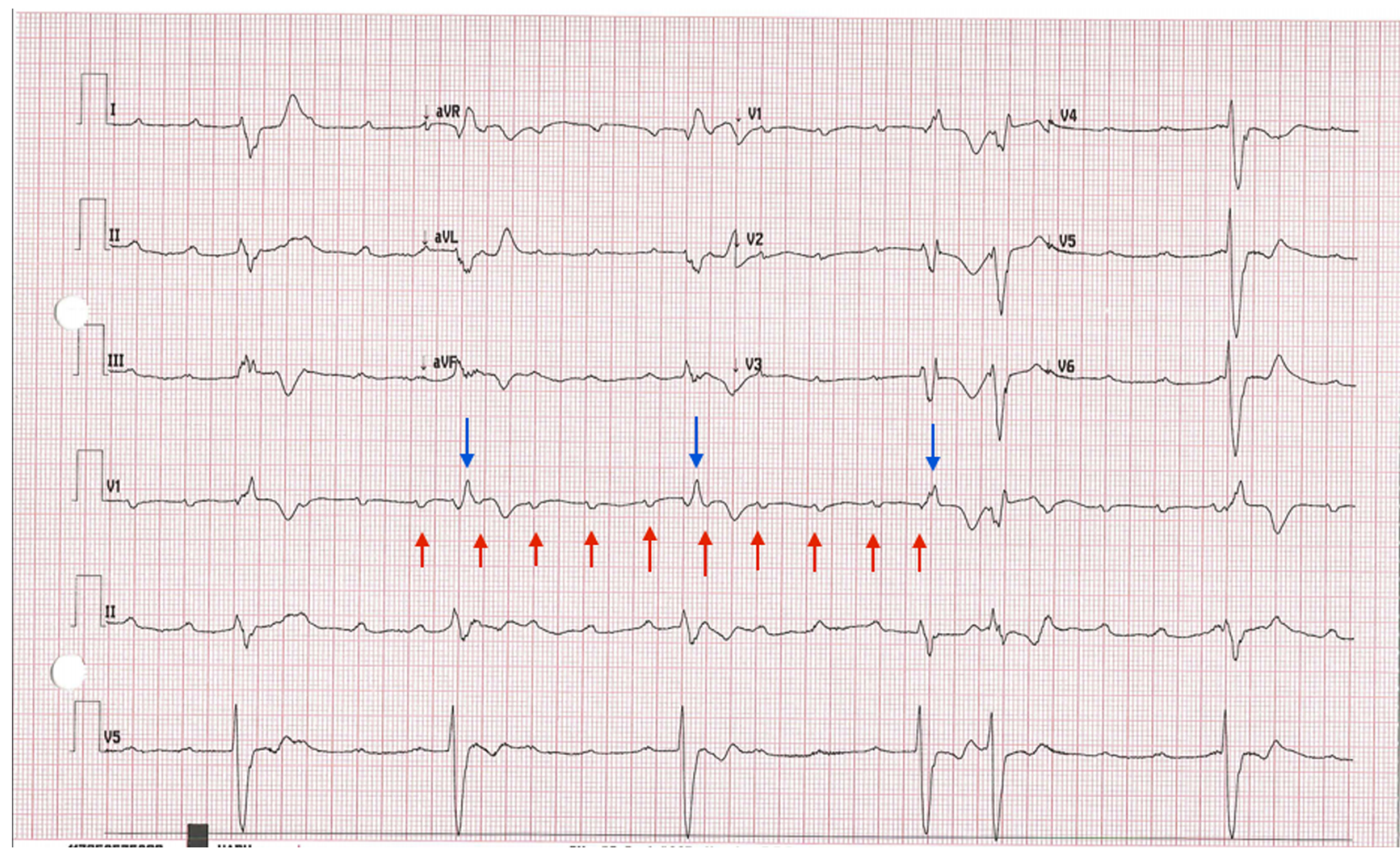


Figure 6. (A) Post-resuscitation ECG showing CHB with RBBB morphology. $\mathrm{P}$ waves are designated by red arrows, while QRS complexes are designated by blue arrows. ST segment elevations are present in the inferior leads with ST segment depressions and $\mathrm{T}$ wave inversions in the anterolateral leads. (B) Subsequent ECG with persistent CHB and RBBB escape rhythm at a slower rate. ST abnormalities have resolved. (C) Native conduction returns and the RBBB noted after resuscitation remains.

worsened, it would have been reasonable to either repeat stress testing as was done or perform anatomic imaging with non-invasive (CT) or invasive coronary angiography.

\section{Imaging in Patients with LBBB}

It has been recognized since 1984 that patients with LBBB but normal coronary arteries may show reversible anteroseptal perfusion defects with exercise scintigraphy using thallium $201{ }^{11}$ Initial studies in dogs who were paced to induce a LBBB showed a reduction in septal flow more prominent at high heart rates. ${ }^{11}$ A subsequent study performed in 1988 on 14 patients with LBBB replicated these results and found that the severity of the septal perfusion defect increased as heart rate increased. It also suggested that the specificity for diagnosing LAD disease in patients with LBBB was only $10 \% .^{12}$ The predictive value of using apical defects to determine LAD disease in patients with LBBB is considered significantly higher. ${ }^{13}$ It is important to note that perfusion defects elsewhere (lateral and inferior wall) are highly predictive of coronary artery stenosis even in

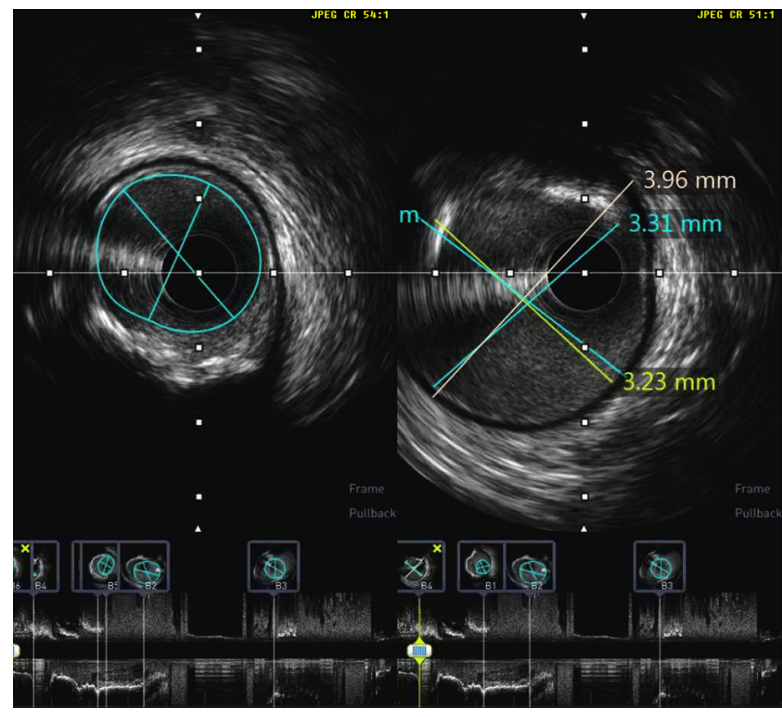

Figure 7. IVUS image obtained to assess the MLA of left main and ostial-proximal LAD.

the presence of LBBB. Similarly, perfusion defects that are present at rest are also unlikely to be an artifact from LBBB ${ }^{14}$ In 1993, adenosine thallium-201 was proven superior to exercise thallium-201 in accurately detecting reversible defects, the presumption being that the perfusion defects did not occur at the lower heart rates achieved with adenosine ${ }^{15}$ A more recent study showed that despite the relatively higher heart rates seen with regadenoson compared to adenosine (but still much lower than with exercise), there was no significant increase in LAD perfusion abnormalities with this stress

\section{C}

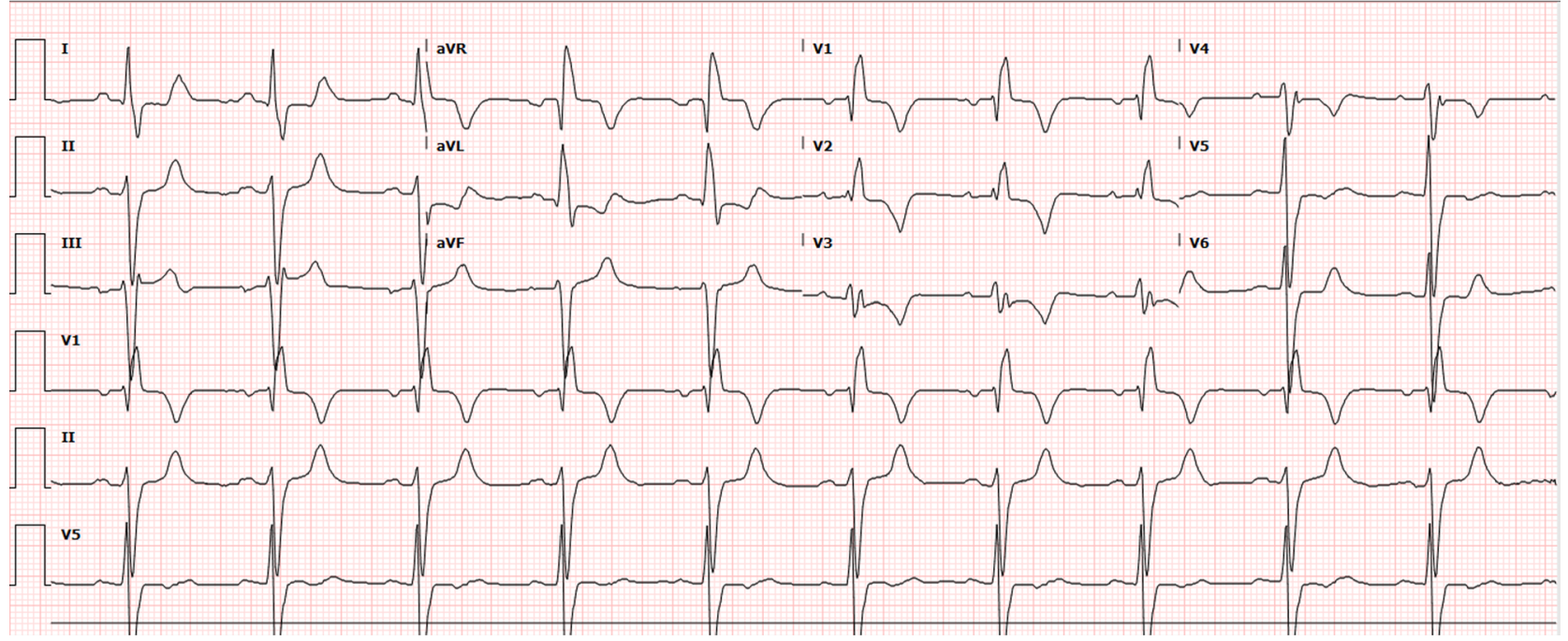

Figure 6. continued. 
agent. ${ }^{16}$ This is in contrast to dobutamine, which like exercise, is associated with a high rate of false-positive septal reversible perfusion defects in LBBB. ${ }^{17}$ Based on these studies, current guidelines recommend vasodilator SPECT MPI as an appropriate initial screening test in patients with LBBB. ${ }^{18,19}$ This patient had a 3-vessel perfusion abnormality on regadenoson MPI, a defect that should not be attributed to a LBBB-related artifact.

\section{Cardiac Events Awaiting CABG}

Cardiac events while awaiting CABG are relatively common (13\%) and have been reviewed extensively with the hope that high-risk patients could be prioritized for early surgery. ${ }^{20}$ These events include rehospitalizations for persistent angina, myocardial infarctions, heart failure exacerbations, and death. Most adverse events happen very early in the waiting process, usually within the first 30 days. $^{20,21}$ The most common, and likely prognostic, risk factor is unstable angina prior to diagnostic angiography. ${ }^{20}$ Other factors associated with death while awaiting $\mathrm{CABG}$ include positive exercise testing, smoking, severe LM or 3-vessel disease, LV dysfunction, NYHA functional class, and triglyceride level. $^{22,23}$ In a large, and frequently cited, Canadian study the death rate was low ( $0.4 \%$ over 14 days) and symptom status and high-risk anatomy were the best predictors of mortality. ${ }^{24}$ Scoring systems have been developed to determine the risk of events while waiting for CABG, using many of the factors already described: unstable angina, LM stenosis, concomitant aortic valve disease, operative risk, LVEF, and male gender. ${ }^{25}$ Our patient likely presented with unstable disease given her symptom progression. She also had a reduced LVEF, LM stenosis, and high triglycerides suggesting that she was a high-risk patient despite her fairly benign initial presentation.

\section{Additional Thoughts}

There are other interesting features present in this case. Our patient did not have angina, but one has to assume that her dyspnea was an angina equivalent. As stated before, patients with DM very commonly present with dyspnea, and the event rate is higher than with typical angina. The cause of her PEA arrest appeared to be hypoxia in the setting of cardiogenic shock and pulmonary edema. What prompted this may have been prehydration in anticipation of coronary angiography. Her sudden deterioration may have also been precipitated by the diagnostic coronary angiogram. It has been long known that intracoronary arterial injections of contrast dye are associated with myocardial ischemia, myocardial dysfunction, and hemodynamic compromise especially in patients with severe CVD. ${ }^{26}$ Alternatively, ischemia in this case may have been due to superimposed coronary spasm, intra-luminal clot, or plaque instability.

The patient developed CHB after resuscitation from a PEA arrest; it is unlikely that she had CHB before the arrest since this was not detected on continuous telemetry. Her coronary perfusion likely decreased despite resuscitative efforts and she must have experienced ischemia of her atrioventricular node. Endogenous adenosine at high concentrations would have been released during ischemia and affected AV conduction resulting in CHB. She was at additional risk for $\mathrm{CHB}$ as she had known infra-nodal conduction disease (RBBB and LBBB). Atropine is generally ineffective in the setting of ischemia-mediated AV block (as demonstrated in our patient) and a trial of aminophylline would have been appropriate. With restoration of blood flow to her AV node after ROSC, her CHB resolved.

The patient developed CHB again in the catheterization lab during right heart catheterization likely due to trauma to the right bundle branch in a patient with pre-existing $\mathrm{LBBB}^{27}$

\section{Disclosure}

Brittain Heindl, Ami E. Iskandrian and Fadi G. Hage declare that they have no conflict of interest.

\section{References}

1. American Diabetes Association. Consensus development conference on the diagnosis of coronary heart disease in people with diabetes: 10-11 February 1998, Miami, Florida. Diabetes Care 1998;21:1551-9.

2. Naka M, Hiramatsu K, Aizawa T, Momose A, Yoshizawa K, Shigematsu S, et al. Silent myocardial ischemia in patients with non-insulin-dependent diabetes mellitus as judged by treadmill exercise testing and coronary angiography. Am Heart $\mathrm{J}$ 1992;123:46-53.

3. Wackers FJT, Young LH, Inzucchi SE, Chyun DA, Davey JA, Barrett EJ, et al. Detection of silent myocardial ischemia in asymptomatic diabetic subjects: The DIAD study. Diabetes Care 2004;27:1954-61.

4. Hage FG, Lusa L, Dondi M, Giubbini R, Iskandrian AE, IAEA Diabetes Investigators. Exercise stress tests for detecting myocardial ischemia in asymptomatic patients with diabetes mellitus. Am J Cardiol 2013;112:14-20.

5. De Lorenzo A, Lima RSL, Siqueira-Filho AG, Pantoja MR. Prevalence and prognostic value of perfusion defects detected by stress technetium-99m sestamibi myocardial perfusion singlephoton emission computed tomography in asymptomatic patients with diabetes mellitus and no known coronary artery disease. Am J Cardiol 2002;90:827-32.

6. Hage FG, Ghimire G, Lester D, Mckay J, Bleich S, El-Hajj S, et al. The prognostic value of regadenoson myocardial perfusion imaging. J Nucl Cardiol 2015;22:1214-21. 
7. Petretta M, Cuocolo A. Screening asymptomatic patients with type 2 diabetes is recommended: Pro. J Nucl Cardiol 2015;22:1225-8.

8. Zellweger MJ, Hachamovitch R, Kang X, Hayes SW, Friedman JD, Germano G, et al. Prognostic relevance of symptoms versus objective evidence of coronary artery disease in diabetic patients. Eur Heart J 2004;25:543-50.

9. Giri S, Shaw LJ, Murthy DR, Travin MI, Miller DD, Hachamovitch $\mathrm{R}$, et al. Impact of diabetes on the risk stratification using stress single-photon emission computed tomography myocardial perfusion imaging in patients with symptoms suggestive of coronary artery disease. Circulation 2002;105:32-40.

10. Sorajja P, Chareonthaitawee P, Rajagopalan N, Miller TD, Frye RL, Hodge DO, et al. Improved survival in asymptomatic diabetic patients with high-risk SPECT imaging treated with coronary artery bypass grafting. Circulation 2005;112:I311-6.

11. Hirzel HO, Senn M, Nuesch K, Buettner C, Pfeiffer A, Hess OM, et al. Thallium-201 scintigraphy in complete left bundle branch block. Am J Cardiol 1984;53:764-9.

12. DePuey EG, Guertler-Krawczynska E, Robbins WL. Thallium-201 SPECT in coronary artery disease patients with left bundle branch block. J Nucl Med 1988;29:1479-85.

13. Matzer L, Kiat H, Friedman JD, Van Train K, Maddahi J, Berman DS. A new approach to the assessment of tomographic thallium201 scintigraphy in patients with left bundle branch block. J Am Coll Cardiol 1991;17:1309-17.

14. Kumar V, Venkataraman R, Aljaroudi W, Osorio J, Heo J, Iskandrian $\mathrm{AE}$, et al. Implications of left bundle branch block in patient treatment. Am J Cardiol 2013;111:291-300.

15. O'Keefe JH Jr, Bateman TM, Barnhart CS. Adenosine thallium201 is superior to exercise thallium-201 for detecting coronary artery disease in patients with left bundle branch block. J Am Coll Cardiol 1993;21:1332-8.

16. Thomas GS, Kinser CR, Kristy R, Xu J, Mahmarian JJ. Is regadenoson an appropriate stressor for MPI in patients with left bundle branch block or pacemakers? J Nucl Cardiol 2013;20:107685.

17. Caner B, Rezaghi C, Uysal U, Tokgozoglu L, Kabakci G, Elahi N, et al. Dobutamine thallium-201 myocardial SPECT in patients with left bundle branch block and normal coronary arteries. J Nucl Med 1997;38:424-7.

18. Wolk MJ, Bailey SR, Doherty JU, Douglas PS, Hendel RC, Kramer CM, et al. ACCF/AHA/ASE/ASNC/HFSA/HRS/SCAI/SCCT/ SCMR/STS 2013 multimodality appropriate use criteria for the detection and risk assessment of stable ischemic heart disease: A report of the American College of Cardiology Foundation Appropriate Use Criteria Task Force, American Heart Association, American Society of Echocardiography, American Society of Nuclear Cardiology, Heart Failure Society of America, Heart Rhythm Society, Society for Cardiovascular Angiography and Interventions, Society of Cardiovascular Computed Tomography, Society for Cardiovascular Magnetic Resonance, and Society of Thoracic Surgeons. J Am Coll Cardiol 2014;63:380-406.

19. Henzlova MJ, Duvall WL, Einstein AJ, Travin MI, Verberne HJ. ASNC imaging guidelines for SPECT nuclear cardiology procedures: Stress, protocols, and tracers. J Nucl Cardiol 2016;23:60639.

20. Koomen EM, Hutten BA, Kelder JC, Redekop WK, Tijssen JG, Kingma JH. Morbidity and mortality in patients waiting for coronary artery bypass surgery. Eur J Cardiothorac Surg 2001;19:260-5.

21. Ray AA, Buth KJ, Sullivan JA, Johnstone DE, Hirsch GM. Waiting for cardiac surgery. Circulation 2001;104:I-92-8.

22. Suttorp MJ, Kingma JH, Vos J, Koomen EM, Tijssen JG, Vermeulen FE, et al. Determinants for early mortality in patients awaiting coronary artery bypass graft surgery: A case-control study. Eur Heart J 1992;13:238-42.

23. Cesena FHY, Favarato D, César LAM, de Oliveira SA, da Luz PL. Cardiac complications during waiting for elective coronary artery bypass graft surgery: Incidence, temporal distribution and predictive factors. Eur J Cardiothorac Surg 2004;25:196-202.

24. Naylor CD, Sykora K, Naylor CD, Jaglal S, Naylor CD, Jefferson S. Waiting for coronary artery bypass surgery: Population-based study of 8517 consecutive patients in Ontario. Canada. Lancet 1995;346:1605-9.

25. Rexius H, Brandrup-Wognsen G, Nilsson J, Odén A, Jeppsson A. A simple score to assess mortality risk in patients waiting for coronary artery bypass grafting. Ann Thorac Surg 2006;81:577-82.

26. Guzman SV, West JW. Cardiac effects of intracoronary arterial injections of various roentgenographic contrast media. Am Heart J 1959;58:597-607.

27. Thomson IR, Dalton BC, Lappas DG, Lowenstein E. Right bundle-branch block and complete heart block caused by the SwanGanz catheter. Anesthesiology 1979;51:359-62.

Publisher's Note Springer Nature remains neutral with regard to jurisdictional claims in published maps and institutional affiliations. 